\title{
El complejo anómalo Oeste de Ceballos, Cuenca Central, Cuba, integración de los métodos no convencionales con la sísmica. Consideraciones sobre la exploración petrolera
}

\author{
Jessica Morales-González ${ }^{1 *}$ [D ; Estenis de la Caridad Martínez-Rojas ${ }^{2}$;

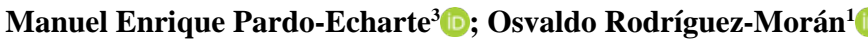

Forma de citar: Morales-González, J.; Martínez-Rojas, E. de la C.; Pardo-Echarte, M.E.; Rodríguez-Morán, O. (2022). El complejo anómalo Oeste de Ceballos, Cuenca Central, Cuba, integración de los métodos no convencionales con la sísmica. Consideraciones sobre la exploración petrolera. Boletín de Geología, 44(1), 135147. https://doi.org/10.18273/revbol.v44n1-2022006

\section{Resumen}

Los métodos no sísmicos y no convencionales constituyen una herramienta de apoyo a los métodos geólogogeofísicos convencionales en la exploración petrolera. La aplicación de estos métodos en la Cuenca Central (yacimientos asociados a la secuencia vulcanógeno-sedimentaria del $\mathrm{K}_{2}$ en trampas estructural-estratigráficas) tuvo buenos resultados, y se cartografió el sector perspectivo Oeste de Ceballos; sin embargo, es indispensable la integración con la sísmica y la información de pozos para validarlo. El objetivo es interpretar la información sísmica existente en este sector con los métodos no sísmicos y no convencionales de exploración. Las líneas sísmicas del sector fueron sometidas a tratamiento de imagen para resaltar los reflectores de interés. El amarre de las líneas sísmicas cercanas con la ubicada sobre el complejo anómalo posibilitó determinar la información geológica de los horizontes de interés. En la parte central existe un levantamiento del tope de las rocas del Cretácico relacionado con sistemas de pliegues. Este alto corresponde a la parte frontal de un pliegue (alto estructural) que coincide con los bordes de la anomalía $\mathrm{K} / \mathrm{Th}$, lo que le confiere un valor especial para la exploración petrolera. Asimismo, los datos litoestratigráficos aportados por el pozo Ceballos 3 muestran que el levantamiento del tope de las rocas del Cretácico corresponde a la presencia de areniscas tobáceas dentro de la cobertura del Arco Volcánico del Cretácico, las cuales se conocen como reservorio en la zona del yacimiento Pina.

Palabras clave: Métodos no sísmicos y no convencionales de exploración; Método sísmico; Cuenca Central; Método gravimétrico; Método magnetométrico.

\section{The anomalous complex Oeste de Ceballos, Central Basin, Cuba, integration between unconventional methods and seismic. Considerations on oil exploration}

\begin{abstract}
Non-seismic and unconventional methods constitute a support tool in oil exploration. The application of these methods in the Central Basin (oil fields associated with the volcano-sedimentary sequence of $\mathrm{K}_{2}$ in structuralstratigraphic traps) had good results, following the mapping of the perspective sector Oeste de Ceballos; however, integration with seismic and well information is essential to validate it. The objective is to interpret the existing seismic information in this sector with the non-seismic and unconventional exploration methods. The seismic lines of the sector were subjected to image treatment to highlight the reflectors of interest. The mooring of the nearby seismic lines with the one located on the anomalous complex, made it possible to determine the geological information of the horizons of interest. In its central part there is a rise of the top of the Cretaceous rocks related to fold systems. This lift corresponds to the front part of a fold (structural lift) that coincides with the edges of the K/Th anomaly, which gives it a special value for oil exploration. Also, the lithostratigraphic data provided by the Ceballos 3 well, shows the rising of the top tuff sandstones within the coverage of the Cretaceous Volcanic Arc, which are known as a reservoir in the area of the Pina oilfield.
\end{abstract}

Keywords: Non-seismic and non-conventional exploration methods; Seismic method; Central Basin; Gravimetric method; Magnetic method.

\footnotetext{
'Departamento de Geociencias, Universidad Tecnológica de La Habana “José Antonio Echeverría”, La Habana, Cuba. (*) jessimoglez@gmail.com,ormoran2016@gmail.com

2Digicupet, Centro de Investigaciones del Petróleo, La Habana, Cuba. esthenis@digi.cupet.cu

${ }^{3}$ Centro de Investigaciones del Petróleo, La Habana, Cuba. pardo@ceinpet.cupet.cu
} 


\section{Introducción}

Las primeras investigaciones exitosas sobre hidrocarburos en la Cuenca Central, Cuba (Figura 1), según Martínez-Rojas et al. (2006), datan de los años 50, cuando compañías norteamericanas descubrieron los yacimientos Jatibonico, Cristales y Catalina; descubiertos en 1954, 1955 y 1956, respectivamente. En la década de los 60, la Cuenca Central fue la mayor región productora de petróleo del país, lo que trajo consigo un desarrollo acelerado de los trabajos de exploración, los cuales se han extendido por más de 50 años de investigación y desarrollo. No obstante, después de la década del 90, con el descubrimiento del yacimiento Pina, no ha habido otro hallazgo de importancia. Este hecho, en parte, se debe a las características geológicas del territorio, como el carácter volcánico de las secuencias presentes, los grandes espesores de ofiolitas y la existencia de fallas y plegamientos que generan difracciones, ondas laterales y ruidos en general, capaces de empeorar la relación señal ruido, enmascarando así, las ondas útiles, por lo que se limita la profundidad de investigación de la sísmica de reflexión, método geofísico por excelencia para la prospección de hidrocarburos. Sin embargo, estudios geológicos realizados indican las posibilidades gasopetrolíferas (Linares et al., 2011) dadas por las frecuentes manifestaciones de asfaltitas presentes en la región.

Los métodos no sísmicos como el gravimétrico, magnético, espectrometría gamma; métodos geomorfológicos y geoquímicos, así como el Complejo Redox, constituyen una herramienta de apoyo a los métodos geofísicos convencionales como el método sísmico, tanto de reflexión como de refracción, que junto con la cartografía geológica posibilitan cartografiar sectores perspectivos para hidrocarburos. Si bien estos métodos brindan, en el estudio de la Cuenca Central, resultados positivos para la determinación de sectores de interés (perspectivos), es indispensable la integración de estos resultados con la sísmica de reflexión. Se tiene como objetivo, entonces, interpretar la información sísmica existente en el sector perspectivo Oeste de Ceballos, que posee un complejo anómalo, a partir de los métodos no sísmicos y no convencionales de exploración.

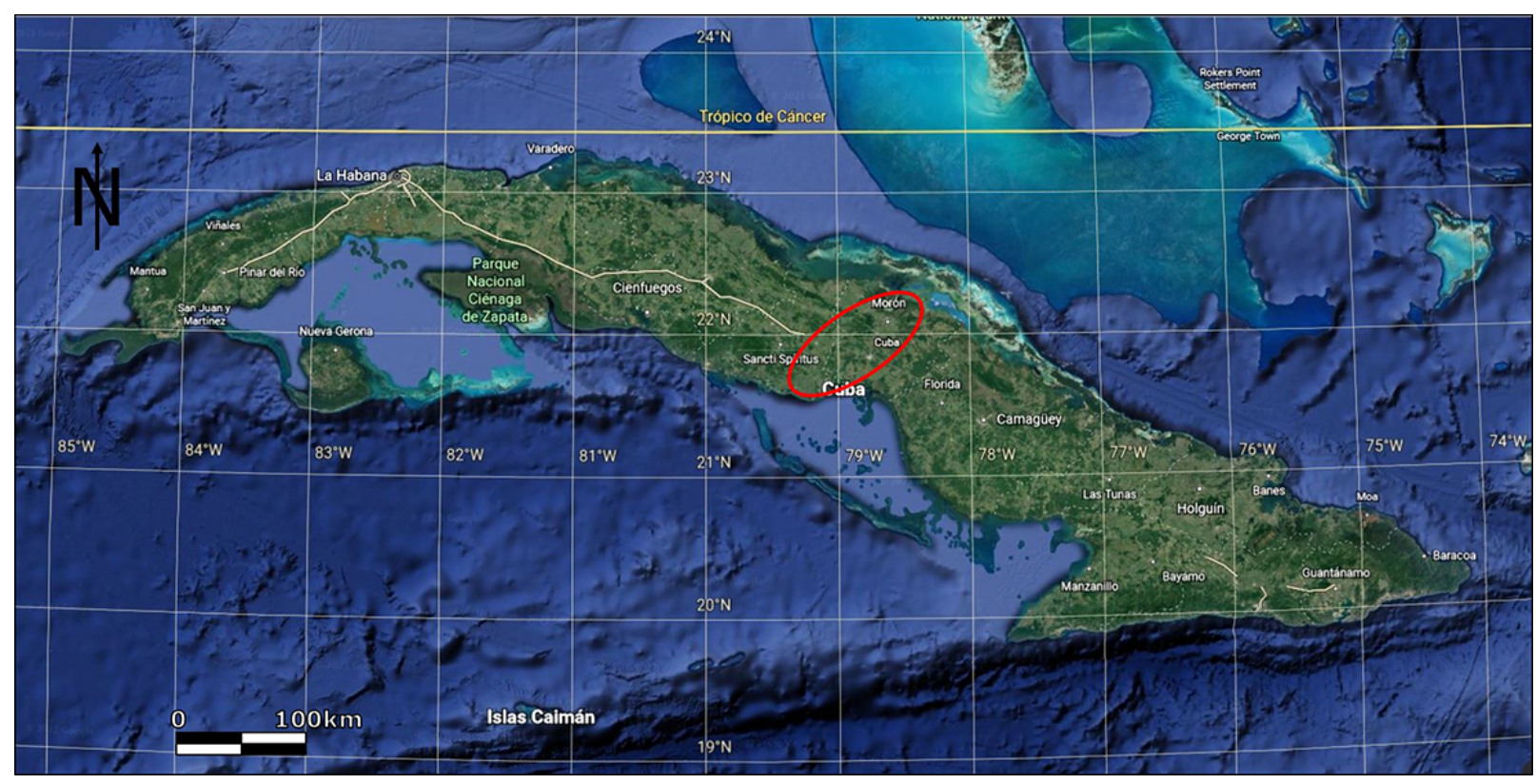

Figura 1. Ubicación de la Cuenca Central de Cuba en las provincias de Ciego de Ávila y Sancti Spiritus (NASA/GSFC, 2020).

\section{Ubicación geográfica y características generales}

La Cuenca Central se ubica en las provincias de Ciego de Ávila y Sancti Spiritus; dentro de este territorio se localizan los yacimientos y prospectos Pina, Brujo, Paraíso, Ceballos. La cuenca se divide de forma geológica en dos semigraben, separados por el alto de Jatibonico. A pesar de su aparente similitud, ambos bloques tienen un comportamiento geólogo-geofísico diferente. En el semigraben localizado al noreste de la Cuenca Central, para el establecimiento de sectores de interés gasopetrolífero, se toma como patrón el modelo generalizado de respuestas (o patrón de respuestas) 
descrito para el yacimiento Pina (Pardo-Echarte et al., 2019). Este se caracteriza por un conjunto de anomalías indicadoras (Figura 2), que consideran los siguientes atributos no sísmicos:

- Máximos gravimétricos locales, a partir de la residual a $500 \mathrm{~m}$ y la primera derivada vertical, los cuales reflejan estructuras positivas por el levantamiento de los volcánicos más densos.

- El yacimiento se encuentra dentro de los límites de las isolíneas del campo aeromagnético reducido al polo, en el intervalo entre -150nT y -115nT, en una anomalía tipo silla de montar.
- Mínimo de la relación K/Th, con máximos locales de U(Ra) mayoritariamente en su periferia.

- Ausencia de anomalías geomórficas.

- Indicaciones anómalas por el Complejo Redox (Figura 3).

Por otra parte, el modelo geológico para el área de estudio, según Martínez-Rojas et al. (2007), considera reservorios del tipo tobas, areniscas y conglomerados tobáceos, asociados al Arco Volcánico Cretácico y su sello en secuencias arcillosas de la cobertura sinorogénica cretácica y postorogénica (Maastrichtiano-Eoceno inferior).

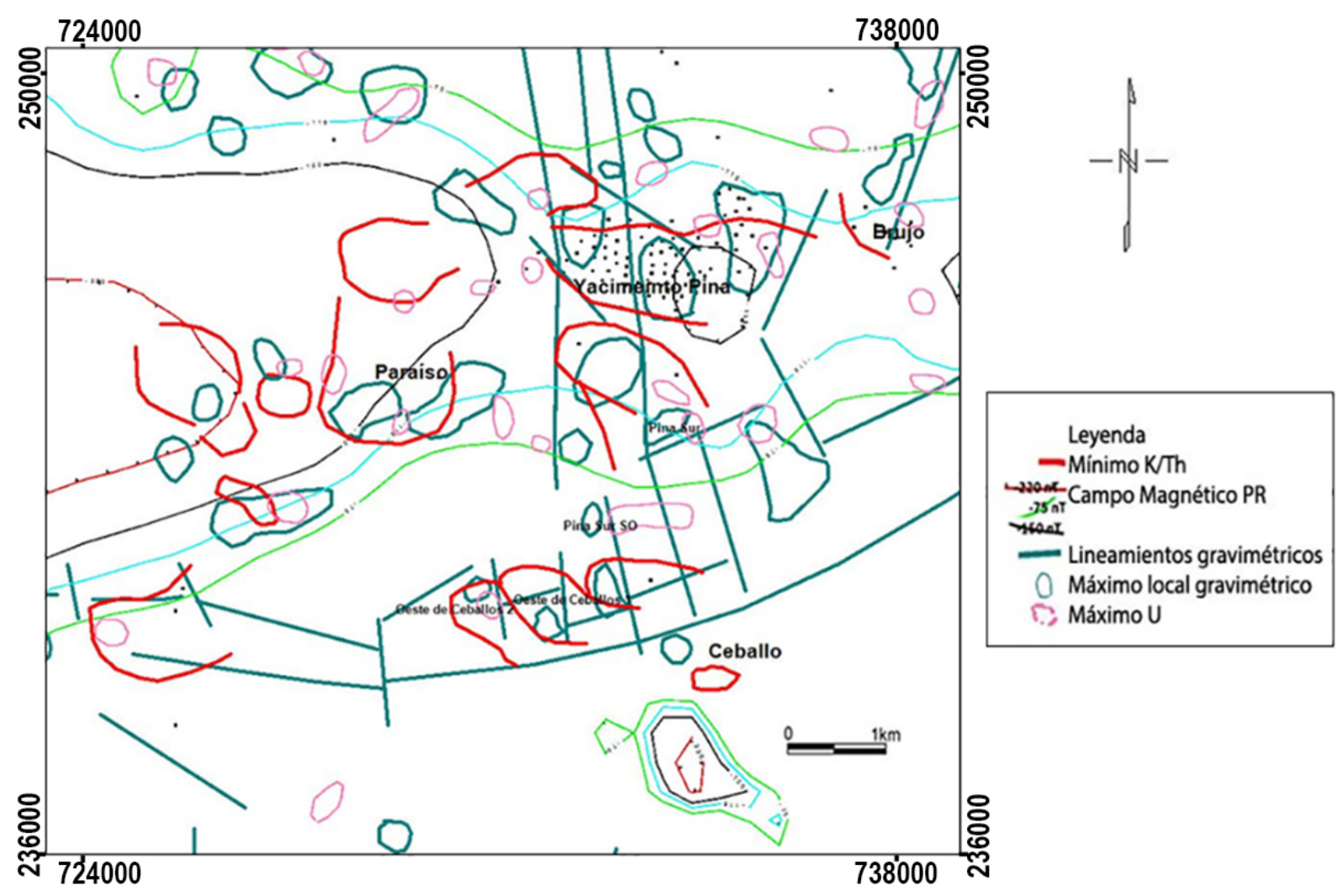

Figura 2. Mapa del patrón de respuestas del yacimiento Pina.

\section{Marco teórico}

\section{Métodos no sísmicos}

Método gravimétrico. El método gravimétrico constituye uno de los métodos de investigación de la estructura geológica de la corteza terrestre, orientado fundamentalmente a la búsqueda y exploración de yacimientos minerales. En el campo gravitatorio tienen lugar las influencias de distribución no homogéneas de densidades en la corteza terrestre, las cuales se manifiestan distintamente, según su cercanía a la superficie, así como su tamaño y posición (Mironov, 1977). Los cuerpos de grandes dimensiones a grandes profundidades producen grandes anomalías en amplitud y área, con variaciones lentas verticales y horizontales. Contrariamente, los cuerpos de pequeñas dimensiones pueden producir anomalías de gran amplitud, pero de pequeña área, con gradientes que denotan gran rapidez en la variación del campo (Peña, 2005). 


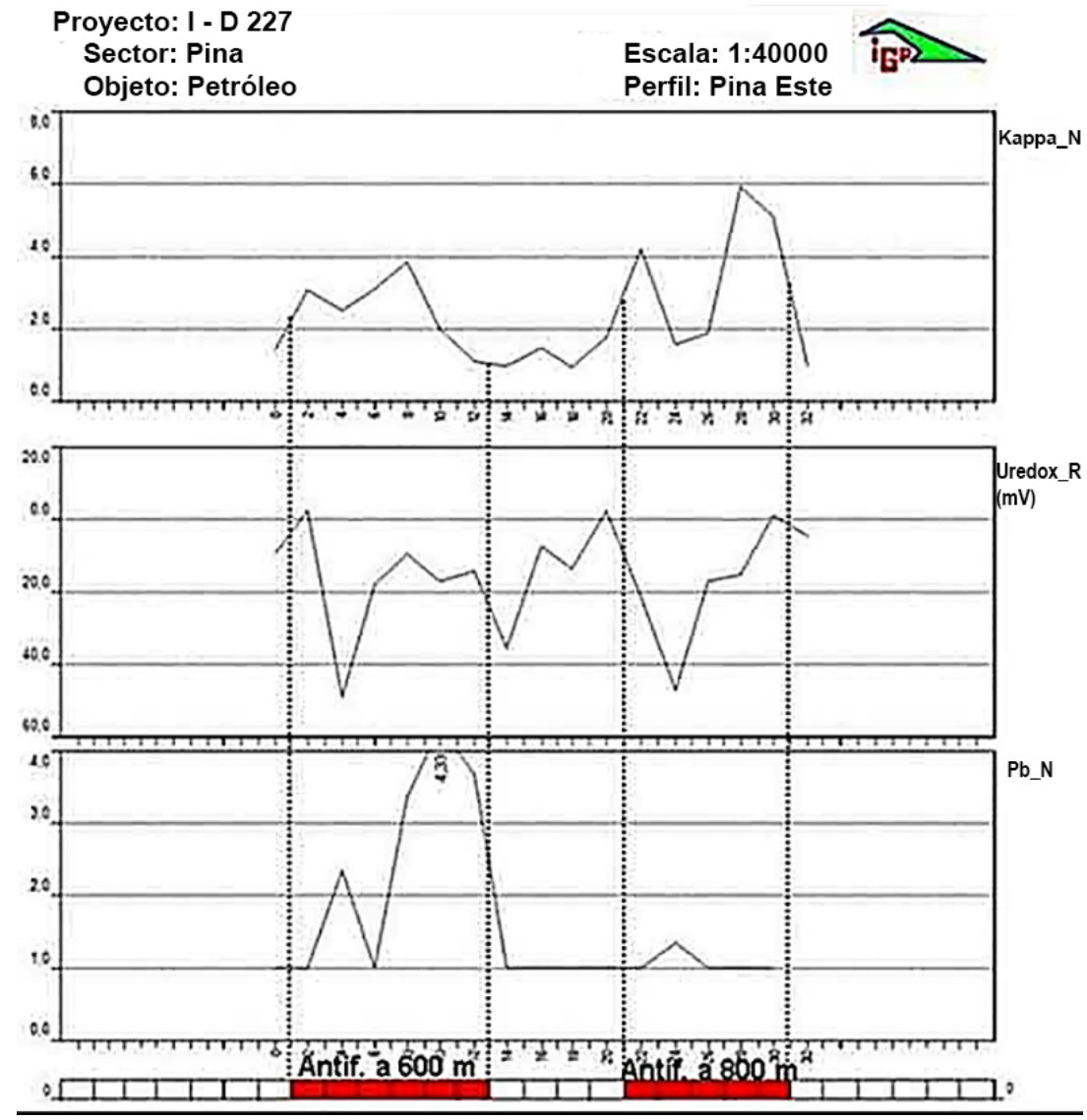

Figura 3. Resultados del Complejo Redox en el yacimiento Pina (Pardo-Echarte et al., 2019).

Dentro de las aplicaciones petroleras, la gravimetría permite la determinación de la geometría de cuencas sedimentarias, donde se determina la "profundidad hasta el basamento", y la evaluación de campos petroleros en apoyo a la exploración sísmica. En la detección de anomalías positivas relacionadas con la presencia de acumulaciones de hidrocarburos, se usan procesamientos como la continuación analítica ascendente (CAA), la derivada vertical de primer orden y la derivada total horizontal. Estos procesamientos permiten cartografiar las anomalías de interés, delimitar sus bordes y también cartografiar los demás elementos tectónicos presentes en el área.

Método magnético. El método magnético se ha empleado para una amplia variedad de problemas relacionados con la cartografía del subsuelo. Normalmente, se utiliza junto con otros métodos geofísicos y con información directa del subsuelo. La relativa facilidad y simplicidad de aplicar el magnetismo lo convierte en una metodología de primera elección, deseable siempre que pueda resolver o contribuir a la solución de estudios del subsuelo (Hinze et al., 2013).

El método magnético se basa en la medición y análisis de perturbaciones o anomalías en el campo magnético terrestre, provocadas por variaciones laterales en la magnetización de materiales del subsuelo. La cartografía de anomalías, junto con la teoría del campo de potencial magnético y las propiedades de magnetización, proporciona una base para aplicar el método magnético en la exploración del subsuelo. La exploración magnética es un método de campo potencial donde la magnitud y dirección del efecto dependen de las posiciones relativas de la fuente de magnetización y del punto de observación, y el contraste de la magnitud y dirección de magnetización, de la fuente con los materiales lateralmente adyacentes.

En muchos sentidos, el método magnético es paralelo al método gravimétrico, pues ambos son métodos de campo potencial que emplean perturbaciones en un campo planetario. Sin embargo, el método magnético 
ofrece varias ventajas sobre el método gravimétrico. Primero, la mayoría de las mediciones se hacen del campo total absoluto con un alto grado de precisión desde un sensor no orientado, lo que simplifica y acelera enormemente las observaciones de campo. En segundo lugar, el rango dinámico de los contrastes de magnetización es mucho mayor que los contrastes de densidad, lo que aumenta la amplitud de las variaciones anómalas. En tercer lugar, la magnetización de los materiales es dipolar en contraste con las propiedades monopolares de la masa. Los efectos de esto son profundos. El resultado es un aumento en la sensibilidad del método magnético a la distancia a la fuente y en la resolución de fuentes individuales (Hinze et al., 2013).

El objetivo de un estudio magnético es investigar la geología del subsuelo sobre la base de anomalías en el campo magnético de la Tierra, como resultado de las propiedades magnéticas de las rocas subyacentes.

Aunque la mayoría de los minerales formadores de roca son efectivamente no magnéticos, ciertos tipos de roca contienen suficientes minerales magnéticos para producir anomalías magnéticas significativas. De manera similar, los objetos ferrosos hechos por el hombre generan anomalías magnéticas (Maldonado, 2019).

En la práctica geológica, este método permite la determinación de la profundidad hasta el basamento, a partir de lo cual se localiza y define la extensión de las cuencas sedimentarias ubicadas encima de este. Asimismo, posibilita la comprensión de la geología regional, con el fin de limitar económicamente los estudios sísmicos en una concesión petrolera, y permite la detección de los efectos magnéticos producidos por la microfiltración de hidrocarburos.

Espectrometría gamma aérea. La espectrometría gamma aérea en la exploración de hidrocarburos se utiliza, convencionalmente, para realizar una cartografía litológica del área de investigación, que complementa la interpretación estructural.

Se tienen en cuenta para ello, la existencia de premisas físico-químico-geológicas como que la descomposición de la arcilla es la responsable de los mínimos de radiación reportados sobre los yacimientos de petróleo; el potasio es lixiviado del sistema hacia los bordes de la proyección vertical del depósito de hidrocarburos, donde precipita y genera un halo de valores altos; el torio permanece relativamente fijo en su distribución original dentro de los minerales pesados insolubles, de ahí que se observen mínimos de la relación K/Th rodeados de máximos sobre los depósitos gasopetrolíferos (Pardo-Echarte et al., 2019).

Morfometría. La morfometría en la prospección de hidrocarburos tiene sus principios en el reconocimiento de áreas favorables para orientar la exploración de estructuras geomorfológicas positivas vinculadas a la formación de levantamientos, que pueden estar relacionados a plegamientos y cabalgamientos (Jiménez et al., 2017). Las anomalías morfométricas asociadas a depósitos de hidrocarburos se fundamentan en las siguientes premisas geológicas:

- Las "Chimeneas Reductoras" son columnas de rocas mineralizadas por encima de los depósitos de hidrocarburos que fueron modificadas por la migración vertical de estos y/o por alguna otra asociación de especies reducidas (iones metálicos), los cuales "se oxidan" por acción microbiana para crear un ambiente reductor.

- Los principales productos de la oxidación microbiana de los hidrocarburos $\left(\mathrm{CO}_{2}\right)$ y de la reducción microbiana del azufre $\left(\mathrm{H}_{2} \mathrm{~S}\right)$ cambian drásticamente el pH/eH del sistema. Los cambios en $\mathrm{pH} / \mathrm{eH}$ resultan en cambios de la estabilidad mineral en precipitación de diversos carbonatos y en la descomposición de las arcillas (como consecuencia, aumentan las concentraciones de sílice y alúmina).

La mineralización secundaria de carbonato de calcio y la silicificación resultan en materiales superficiales más densos y resistentes a la erosión, por lo que posibilitan la formación de anomalías geomórficas y máximos de resistividad (Pardo-Echarte et al., 2019).

Complejo Redox. Las acumulaciones de hidrocarburos presentan generalmente escapes o microfiltraciones, predominantemente verticales. La expresión superficial de la microfiltración de hidrocarburos adopta variadas formas, las cuales determinan el desarrollo de un semejante número de métodos de detección tanto directos (geoquímica de gases de hidrocarburos) como indirectos (Complejo Redox).

En la exploración petrolera, el Complejo Redox posibilita el reconocimiento y la cartografía de la proyección vertical de la ocurrencia con la estimación, de manera indirecta y aproximada, de la profundidad de la ocurrencia, el posible tipo de trampa estructural, la calidad del hidrocarburo y el volumen de la ocurrencia, a partir de los resultados de la cartografía. No obstante, el Complejo Redox presenta varias limitaciones, 
por ejemplo: la no detección de varios niveles de hidrocarburos superpuestos (incluye casquetes de gas); el fracaso si la acumulación carece de sello o si el mismo está muy fracturado. Además, se ve afectado por la presencia de zonas reductoras superficiales de diversa naturaleza, por el predominio de la respuesta litológica de la susceptibilidad magnética y la reflectancia espectral y por la falta de respuesta frente a una cobertura transportada reciente (menor de 10 años) (Pardo-Echarte y Rodríguez, 2016).

La interpretación cualitativa del Complejo Redox comprende la identificación del incremento en el suelo de los elementos metálicos constituyentes básicos que migran desde la fuente, con respecto al fondo local de los elementos químicos característicos de los suelos residuales (cortezas de intemperismo), los cuales pueden estar afectados por diversos tipos de alteraciones vinculadas con la propia fuente. Esto es válido para los atributos físicos medidos como el potencial redox (Uredox), la kappametría (Kappa) y la reflectancia espectral (RE), donde el problema de la interpretación cualitativa considera la identificación, en términos de gradientes (incrementos o decrementos respecto al fondo local), del comportamiento de cada atributo ante la contribución anómala de los elementos metálicos constituyentes básicos provenientes de la fuente. De ahí que resulte pertinente tratar con atributos reducidos o normalizados con respecto al fondo local (en dependencia de que la distribución característica para el atributo medido sea normal o lognormal) (Pardo-Echarte y Rodríguez, 2016).

\section{Método sísmico}

El método sísmico de reflexión es el método que lidera la exploración petrolera; se excita energía elástica y se reciben sus "ecos" (reflexiones) procedentes del subsuelo. Este método requiere una fuente sísmica de energía como dinamita, cañones de aire o vibroseis, que genere una señal que se transmite a través del medio elástico hacia la profundidad; después de un cierto intervalo, esta energía es reflejada por cualquier discontinuidad física o por estratos subyacentes, provocando el movimiento de un transductor electromecánico (detector) o piezoeléctrico. La señal de este detector es amplificada y se registra en un equipo especializado. Observando el tiempo que toma una reflexión para arribar a un receptor, es posible estimar la profundidad de la característica que ha generado la reflexión. Generalmente, permite investigar profundidades de unos $10 \mathrm{~km}$ e incluso en ciertos trabajos puede alcanzar mayores profundidades.
En dependencia de la profundad de estudio, la prospección sísmica es una herramienta de investigación poderosa que brinda la posibilidad de estudiar con buena resolución desde los primeros metros del terreno (sísmica de alta resolución o sísmica superficial, shallow seismic) hasta varios kilómetros de profundidad (sísmica profunda, deep seismic). A partir del estudio de las distintas formas de onda y sus tiempos de trayecto, se consigue obtener imágenes del subsuelo que luego se relacionan con las capas geológicas (secciones sísmicas, campos de velocidades) (Ceballos, 2020).

\section{Metodología}

\section{Materiales}

1. Informes geológicos, geofísicos, geoquímicos y geomorfológicos de la Cuenca Central, Cuba.

2. Informes y publicaciones temáticos de investigación geológica y petrolera en la región.

3. Mallas de datos del campo gravimétrico y aeromagnético a escala 1:50000 del territorio (Mondelo y Sánchez, 2011).

4. Mapa geológico digital de Cuba (IGP) a escala 1:100000 (Colectivo de autores, 2010).

5. Mapas digitales de las manifestaciones de hidrocarburos (Colectivo de autores, 2008) y de los pozos petroleros de la República de Cuba (Colectivo de autores, 2009).

6. Líneas sísmicas postsuma adquiridas por CUPET (García y Valdés, 2004).

\section{Premisas de exploración}

En el reconocimiento de las zonas favorables para hidrocarburos, los métodos geofísico-geoquímicos no convencionales como el Complejo Redox (potencial redox (Uredox), la kappametría (Kappa) y la reflectancia espectral (RE)), la magnetometría, la espectrometría la gamma y la morfometría se aplicaron a partir de una estrategia de exploración, en la que se integran con los métodos sísmicos y gravimétricos; se consideró la elaboración de los siguientes escenarios (Pardo-Echarte et al., 2019):

- Sísmica: levantamientos estructurales de las rocas volcánicas.

- Gravimetría: máximos locales positivos (revelados, como regla, en la primera derivada vertical), debido al levantamiento de las rocas volcánicas más densas, coincidentes o próximos a 
los levantamientos sísmicos.

- Aeromagnetometría: tendencia regional del campo magnético reducido al polo (RP) entre $-220 \mathrm{y}$ -75nT (posible parte más levantada de la cuenca).

- Espectrometría gamma aérea: mínimos de la relación $\mathrm{K} / \mathrm{Th}$ y mayoritariamente en su periferia, máximos locales de U(Ra).

- Morfometría: sutiles máximos locales positivos del relieve residual a $500 \mathrm{~m}$ que, como regla, no se observan en el caso tratado.

Con base en la integración de esos escenarios se obtienen complejos anómalos que son reconocidos en tierra por un perfil del Complejo Redox.

\section{Resultados}

El sector Oeste de Ceballos (Oeste de Ceballos 1 y Oeste de Ceballos 2) (Figura 4) reproduce, en su totalidad, el patrón de respuestas del yacimiento Pina, al ser este de gran interés por estar vinculado a la extensión de la falla Cristales.

Los perfiles de Complejo Redox (Figuras 5 y 6) confirmaron la posible presencia de hidrocarburos en la profundidad en las zonas Oeste de Ceballos 1 y 2, a partir de la existencia de incrementos mayores o iguales a dos veces el fondo de los contenidos correlacionados de $\mathrm{V}$ y Ni (elementos indicadores de hidrocarburos), coincidentes con incrementos de Fe, Pb y Zn. También, se observa (el sector señalado en rojo) (Figuras 5 y 6) una correlación entre máximos de la susceptibilidad magnética con incrementos de los elementos químicos (V y Ni), aunque en la zona de Oeste de Ceballos 1 se ve que existen máximos de potencial redox, asociados a posibles escapes gaseosos. Desde el punto de vista geoquímico (contenidos de $\mathrm{V}$ y $\mathrm{Ni}$ ), el intervalo anómalo más contrastante es el de Oeste de Ceballos 2, que supera más de tres veces el fondo.

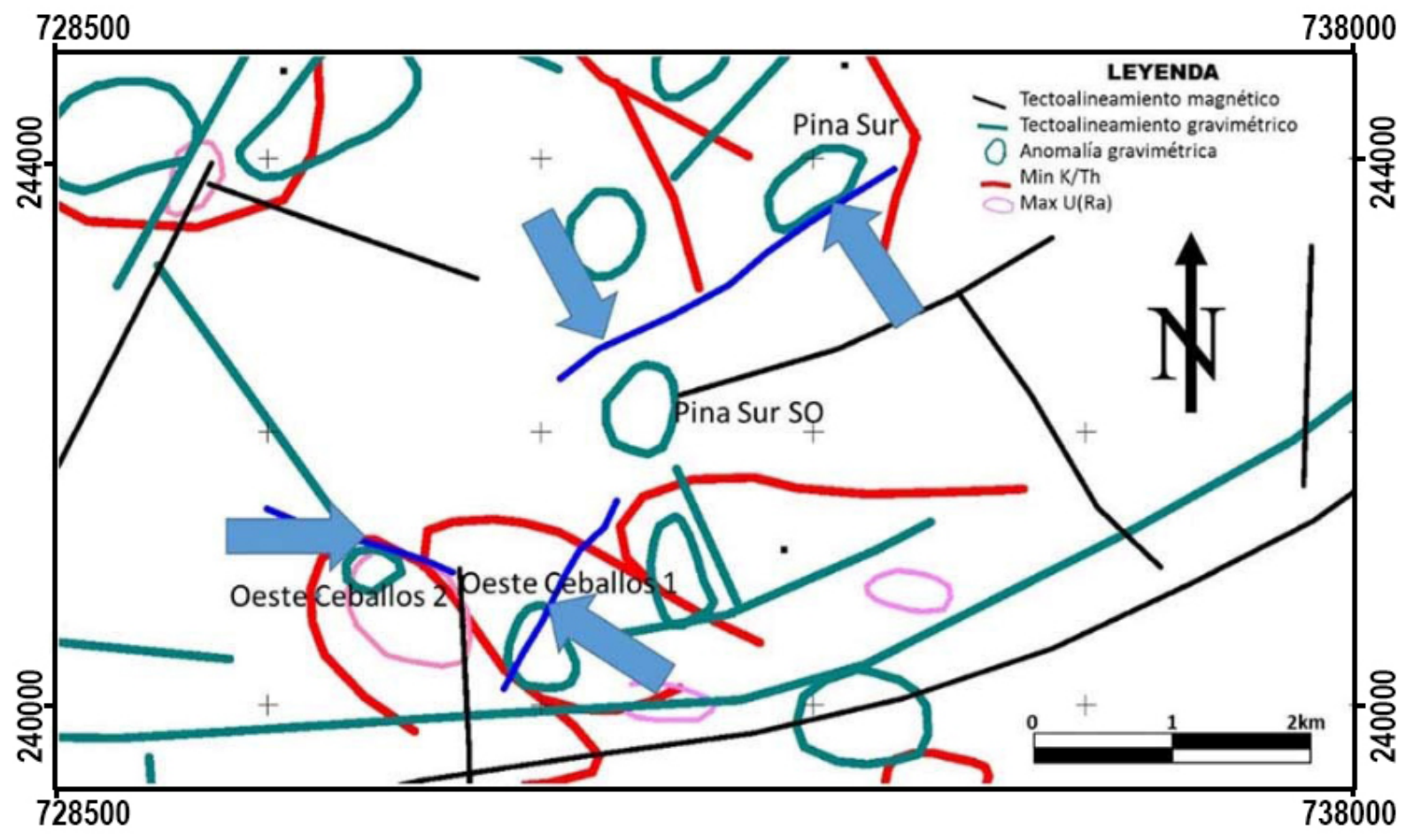

Figura 4. Ubicación de los perfiles del Complejo Redox medidos (líneas azules) y señalización (con una flecha azul) de los intervalos anómalos, durante la Campaña I (Pardo-Echarte et al., 2019). 
EI complejo anómalo Oeste de Ceballos, Cuenca Central, Cuba, integración de los métodos no convencionales con la sísmica. Consideraciones sobre la exploración petrolera

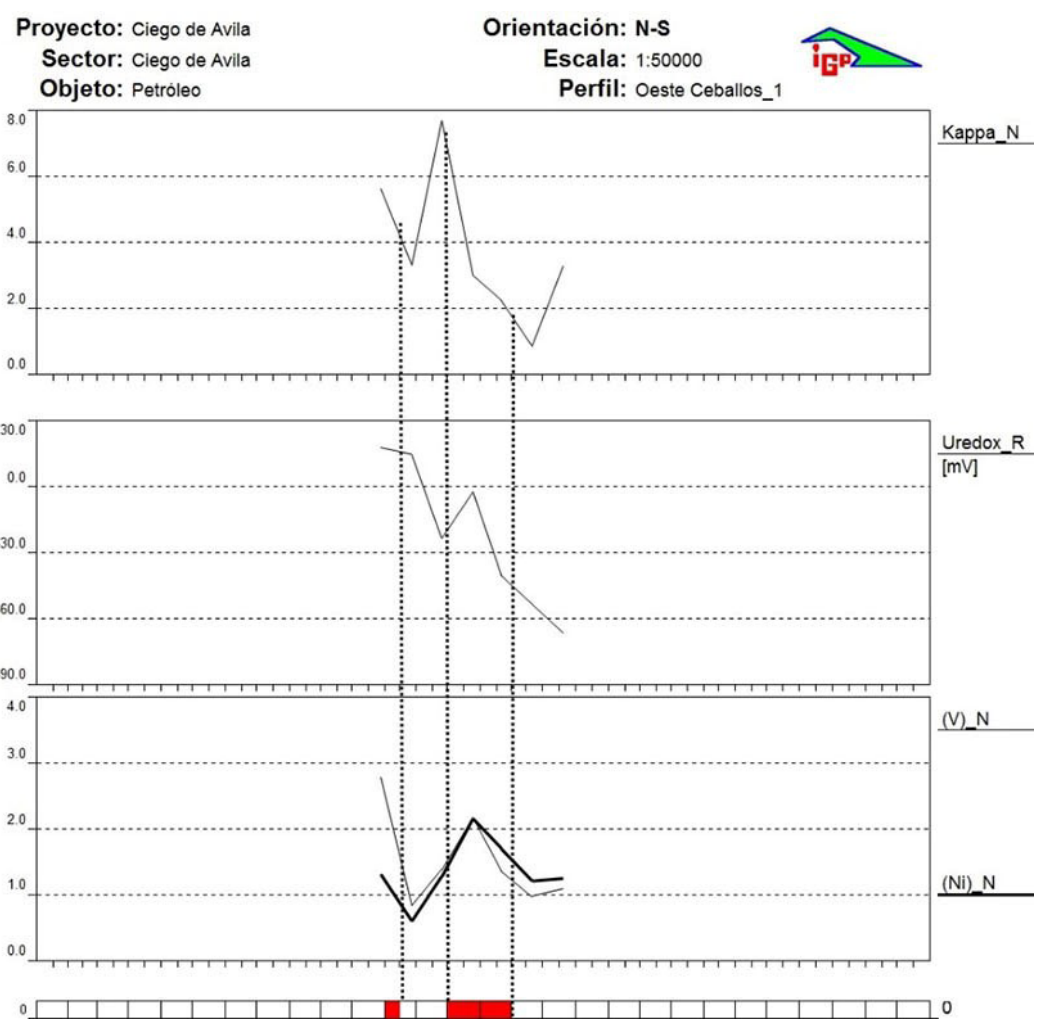

Figura 5. Perfil de medición Oeste de Ceballos 1. La distancia entre puntos de medición es orientativa (Pardo-Echarte et al., 2019).

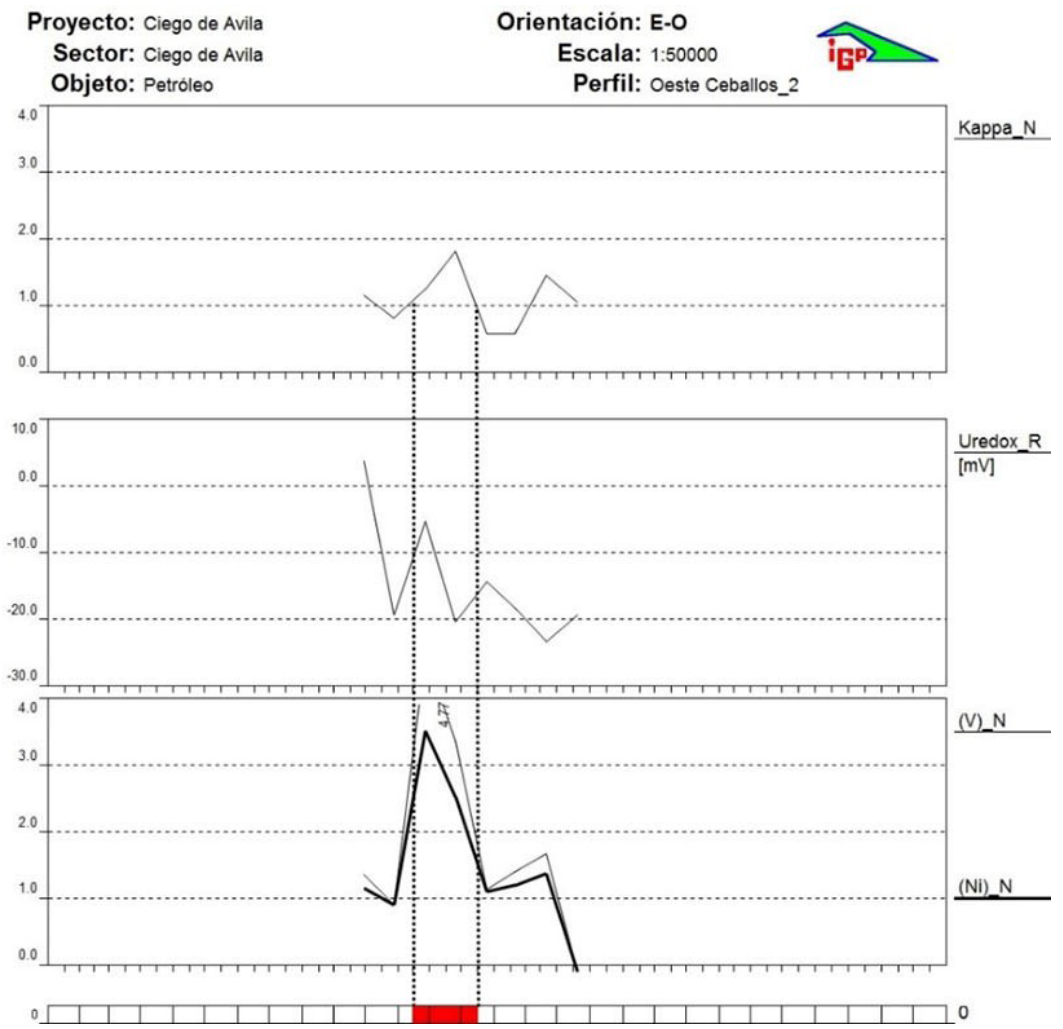

Figura 6. Perfil de medición Oeste de Ceballos 2. La distancia entre puntos de medición es orientativa (Pardo-Echarte et al., 2019). 
Sobre el sector perspectivo Oeste de Ceballos (Figura 7) se ubica la línea sísmica 11 (Sterling y Martínez-Rojas, 2018) (Figura 8). La longitud de la línea no alcanza una buena profundidad de estudio y se observan ruidos a los laterales que limitan la interpretación. Sobre esta línea o cerca no se localizan pozos, entonces, para identificar los posibles contactos geológicos fue necesario hacer un cruce entre las líneas 13 (Sterling y Martínez-Rojas, 2018) (paralela a la línea 11) y 5 (Figura 10) (Sterling y Martínez-Rojas, 2018) (perpendicular a las otras dos), las que a su vez se amarraron con los pozos Agustín 3 y Ceballos 3 (Figura 9).

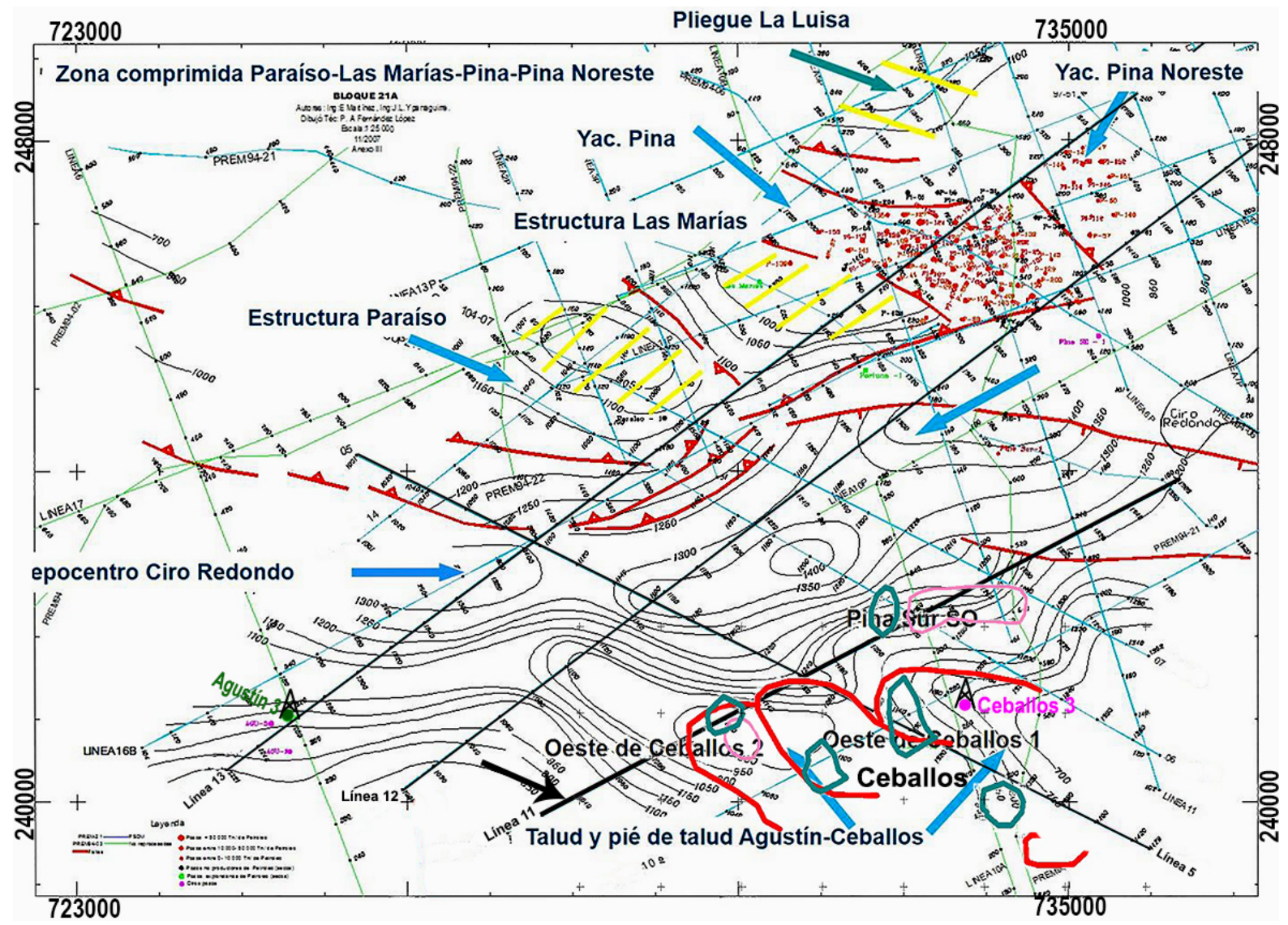

Figura 7. Complejo anómalo de las zonas perspectivas Oestes de Ceballos 1, 2 y Pina sur SO sobre el mapa estructural, por la base del paquete de reflectores sísmicos de edad $\mathrm{K}_{2}^{\mathrm{cp}-\mathrm{m}}$, asociado a las rocas productoras en el área Pina y a las manifestaciones de hidrocarburos en los pozos Paraíso, Agustín y Ceballos. Escala 1:25000, modificado de Martínez-Rojas et al. (2006).

Las líneas sísmicas 2D presuma en tiempo (PSTM) localizadas en la Cuenca Central de Cuba fueron procesadas por el Departamento de Procesamiento de Datos de DIGICUPET con el software GeoEast V2.6.3 de procedencia China. La secuencia de procesamiento empleada consistió en la etapa de preprocesamiento, seguido de una migración presuma en tiempo y del posprocesamiento. Se obtuvieron los sismogramas con el resultado del preprocesamiento (sin migración) y de la migración presuma en tiempo, las sumas migradas sin pos-procesamiento y con posprocesamiento, así como, las velocidades de suma y migración.

El cálculo de las profundidades de yacencia de los diferentes horizontes sísmicos correlacionados y calibrados, según las columnas litoestratigráficas de los pozos Agustín 3 y Ceballos 3 (Figura 9), se realizó a partir de las velocidades de la migración PSTM de los CDP más cercanos a los pozos. También se graficaron las velocidades de intervalo y las velocidades medias (a partir de los pares de la velocidad RMS (módulo PostMigVField), el cual emplea la fórmula de Dix), las primeras para una mejor definición de los diferentes paquetes rocosos y las segundas para comparar con los resultados del VSP del pozo Ceballos 1 en las proximidades de la línea 10A en cruce con la línea 5 . También, posibilitó el cruce de las líneas sísmicas 13 y 12 con la 5 y, a su vez, la línea 5 con la 11, para obtener las profundidades, las que fueron amarradas con los datos de los pozos Agustín 3, en el caso de la línea 13, y la línea 5 con el pozo Ceballos 3 (Figura 10). 


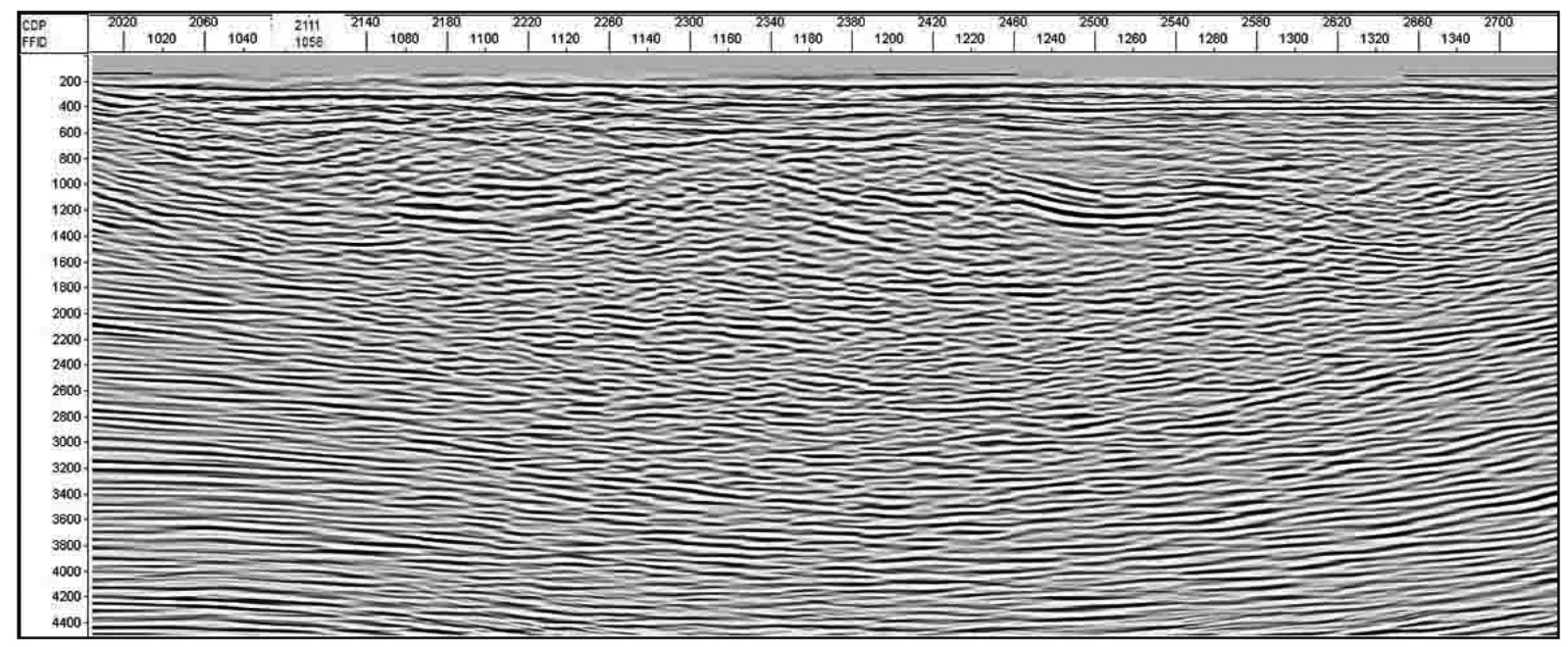

Figura 8. Línea sísmica PSTM 11 con tratamiento de imagen (Martínez-Rojas y Morales-González, 2020).

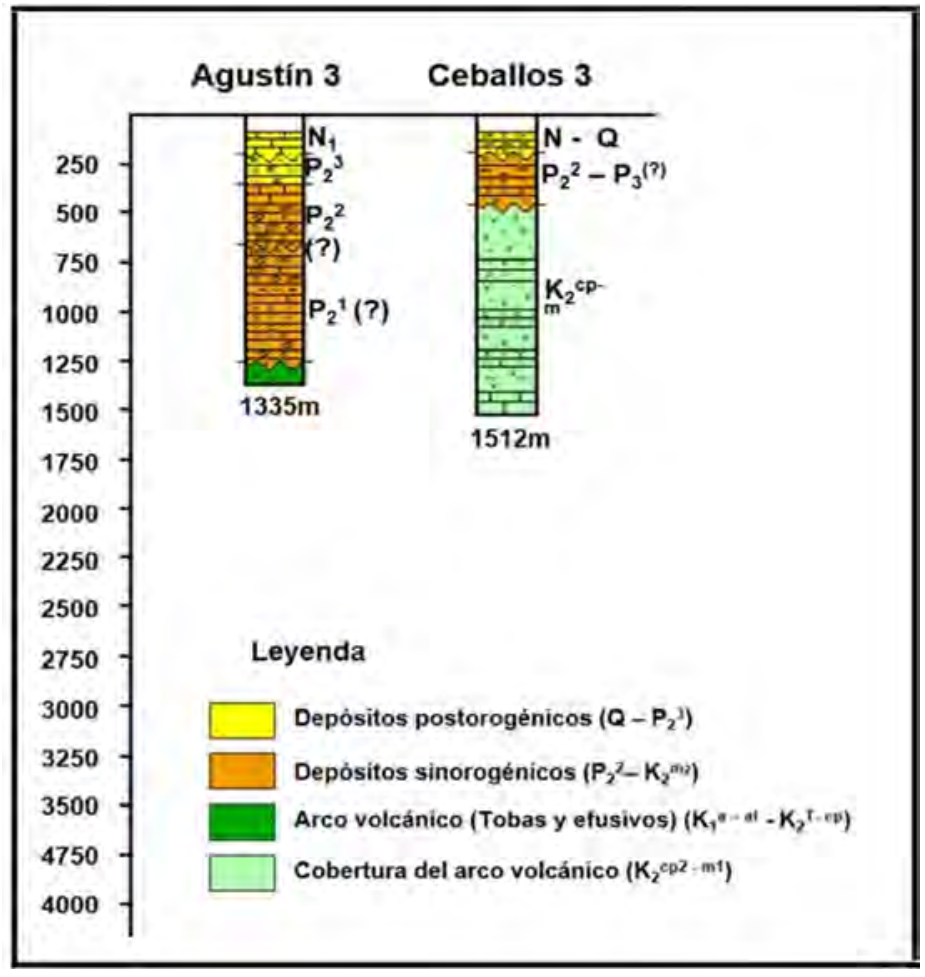

Figura 9. Columnas litoestratigráficas de los pozos Agustín 3 y Ceballos 3.

\section{Discusión}

A partir del cruce de las líneas sísmicas y del amarre con los pozos Agustín 3 y Ceballos 3, se obtuvieron los horizontes que corresponden a la base del Eoceno superior-Mioceno y el tope del Cretácico en la línea 5 (Figura 10). Por debajo del tope de las rocas del Cretácico, la imagen no muestra una disposición del cuadro sísmico favorable, lo que imposibilita la ubicación de la base, aunque existe un estimado a partir del mapa estructural (Figura 7) por la base del paquete de reflectores sísmicos de edad $\mathrm{K}_{2}{ }^{\mathrm{cp}-\mathrm{m}}$.

El amarre de la línea 13 con el pozo Agustín 3 ubicó la base del Neógeno a $330 \mathrm{~m}$ y la base del Eoceno inferior y medio (?) a $1250 \mathrm{~m}$; el pozo finalizó a 1335 $\mathrm{m}$. Mientras que entre los 0,7 y $0,8 \mathrm{~ms}$ se encuentra una discordancia litoestratigráfica entre las secuencias del 
Paleógeno (?), Paleoceno inferior y medio y las rocas del Arco Volcánico del Cretácico (?).

Los horizontes determinados tanto en la línea 13 como en la 5 fueron identificados en la 11 mediante el amarre de estas (Figura 11). La línea 11 muestra en la parte central un evento asociado a un levantamiento relacionado con un sistema de pliegues (Figura 12). Se considera que este alto corresponde a la parte frontal de un pliegue que coincide con los bordes de la anomalía de la relación potasio-torio (Figura 7).

Según los datos litoestratigráficos aportados por el pozo Ceballos 3, el levantamiento del tope de las rocas del Cretácico corresponde a la presencia de areniscas tobáceas dentro de la cobertura del Arco Volcánico del Cretácico (Figura 11), las cuales se conocen como reservorio en la zona del yacimiento Pina.

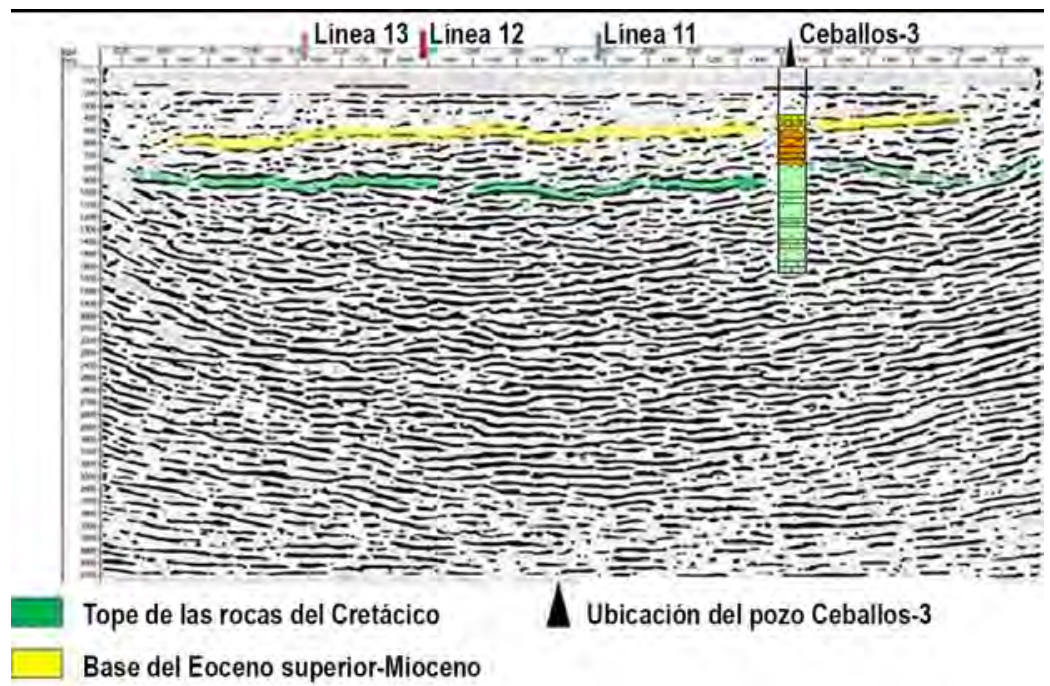

Figura 10. Ubicación del tope de las rocas del Cretácico y de la base del Eoceno superior-Mioceno sobre la línea sísmica 5 PSTM ya tratada (Martínez-Rojas y Morales-González, 2020).

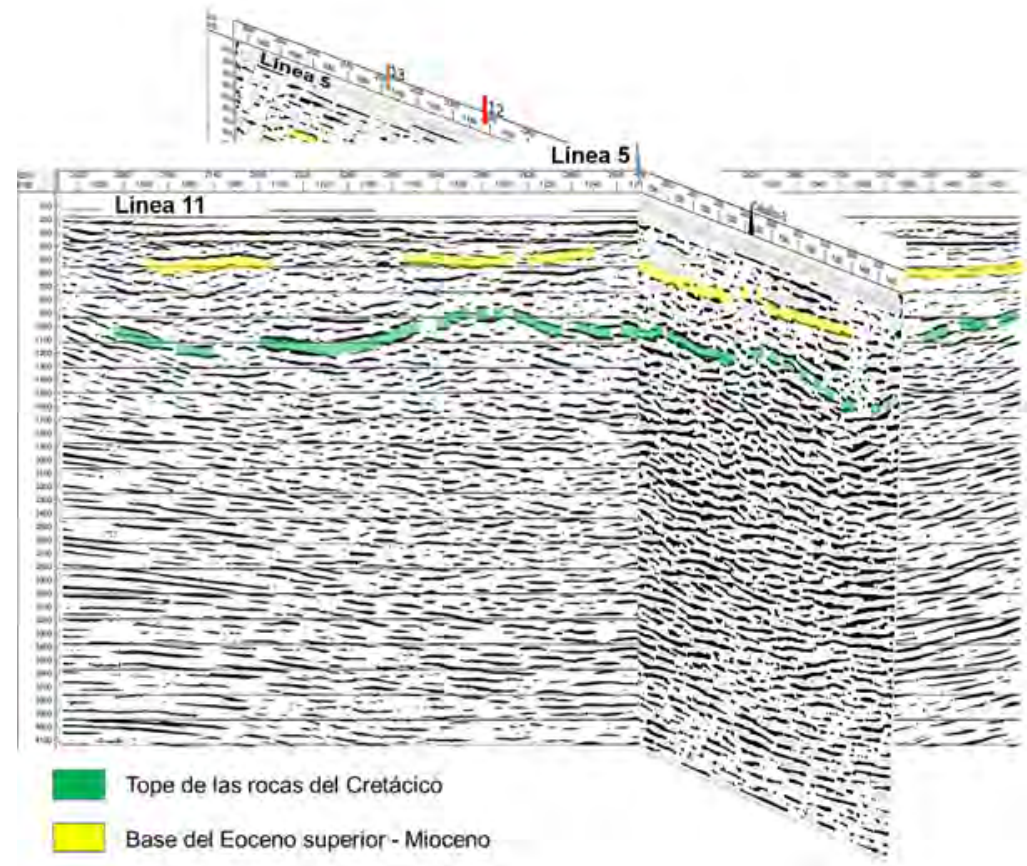

Figura 11. Cruce entre las líneas símicas (interpretadas a partir del tratamiento de pixeles) 5 con 11 (Martínez-Rojas y MoralesGonzález, 2020). 


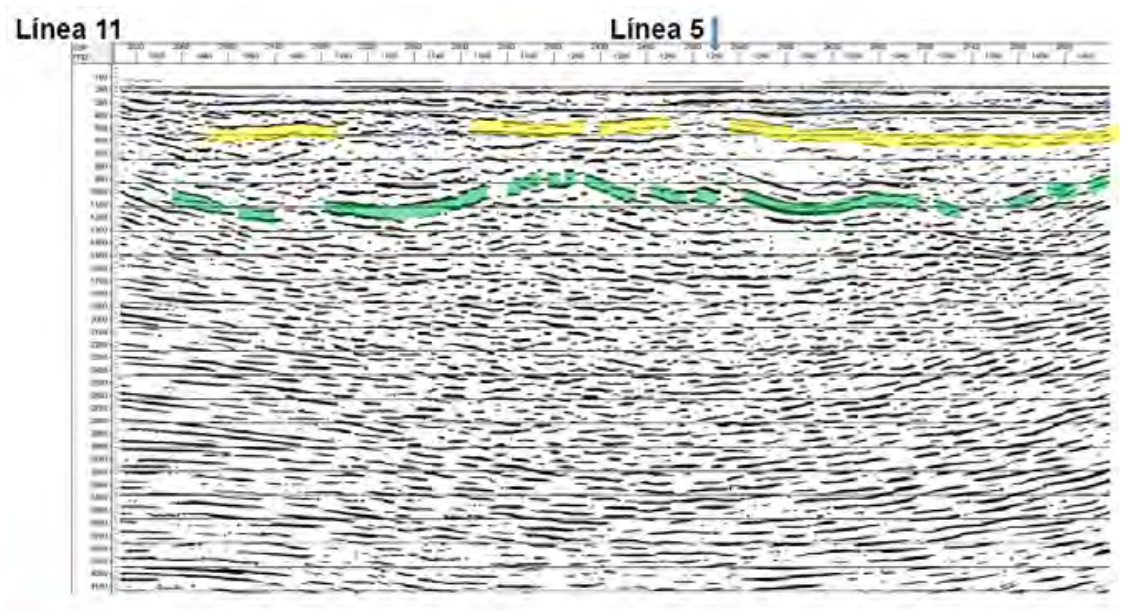

Tope de las rocas del Cretácico

Base del Eoceno superior - Mioceno

Figura 12. Interpretación de la línea sísmica 11 a partir del tratamiento de pixeles (Martínez-Rojas y Morales-González, 2020).

\section{Conclusiones}

El amarre de las líneas sísmicas cercanas al sector Oeste de Ceballos con la línea ubicada sobre el complejo anómalo posibilitó determinar la litología y la edad de los horizontes de interés. En la parte central, se revela un evento asociado a un levantamiento del tope de las rocas del Cretácico, posiblemente relacionado con un sistema de pliegues. Se considera que este alto corresponde a la parte frontal de un pliegue que coincide con los bordes de la anomalía de la relación potasio-torio, lo que le confiere un mayor valor para la exploración petrolera.

Los datos litoestratigráficos aportados por el pozo Ceballos 3 muestran que el levantamiento del tope de las rocas del Cretácico corresponde a la presencia de areniscas tobáceas dentro de la cobertura del Arco Volcánico del Cretácico, las cuales se conocen por funcionar como reservorio en la zona del yacimiento Pina.

El empleo de los métodos no sísmicos posibilitó la cartografía del sector perspectivo Oeste de Ceballos, el que, validado por la sísmica, mostró resultados favorables para la posible existencia de una acumulación de hidrocarburos en el referido sector.

\section{Agradecimientos}

Los autores desean agradecer al Centro de Investigación del Petróleo por permitir el uso de la información necesaria para el desarrollo de la investigación.
También, agradecen al Dr. Juan Guillermo LópezRivera, Dr. Reinaldo Rojas-Consuegra, Dr. Evelio Linares-Cala, MSc. Orelvis Delgado-López y al especialista Héctor Grabosqui, por su siempre oportuna colaboración.

\section{Referencias}

Ceballos, D. (2020). Diseño de adquisición sísmica 2D en la zona costa afuera del Bloque $\mathrm{D}$, de la Zona Económica Exclusiva de Cuba (ZEE), Bahía de Nipe, provincia de Holguín. Tesis, Universidad Tecnológica de La Habana "José Antonio Echeverría", Cuba.

Colectivo de autores (2008). Mapa Digital de las Manifestaciones de Hidrocarburos de la República de Cuba a escala 1:250000. Centro de Investigaciones del Petróleo.

Colectivo de autores (2009). Mapa Digital de los Pozos Petroleros de la República de Cuba a escala 1:250000. Centro de Investigaciones del Petróleo.

Colectivo de autores (2010). Mapa Geológico Digital de Cuba a escala 1:100000. Instituto de Geología y Paleontología.

García, R.; Valdés, P. (2004). Reporte de las investigaciones geológicas sobre las líneas sísmicas en el sector noroccidental de la Cuenca Central, Bloque 21. Provincia Ciego de Ávila. Centro de Investigaciones del Petróleo. 
Hinze, W.; Von Frese, R.; Saad, A. (2013). Gravity and Magnetic Exploration. Principles, Practices, and Applications. Cambridge University Press.

Jiménez, L.; Cruz, R.; Pérez, Y.; Valdivia, C.; Rodríguez, O.; Pérez, M.V. (2017). Métodos geológicos y geomorfológicos para la exploración petrolera en la región centro-oriental de Cuba. Minería y Geología, 33(1), 13-25.

Linares, E.; García, D.E.; Delgado, O.; López, J.G.; Strazhevich, V. (2011). Yacimientos y manifestaciones de hidrocarburos de la República de Cuba. Centro de Investigaciones del Petróleo.

Maldonado, Y. (2019). Método magnético. https:// geologiaweb.com/geofisica/metodo-magnetico

Martínez-Rojas, E.; Toucet, S.; Sterling, N.; Yparraguirre, J.L. (2006). Informe sobre la reinterpretación geólogo-geofísica y evaluación estructural del Bloque 21. Reinterpretación sísmica terrestre 2D. Centro de Investigaciones del Petróleo.

Martínez-Rojas, E.; Iparraguirre, J.L.; Sterling, N.; Prol, J.L.; Gómez, R.; Capote, C.; Cruz, R.; García, R.; Toucet, S.; Amador, H.; López, O.; Perera, C. (2007). Configuración tectono-estructural de la región noreste de Cuenca Central: una visión preliminar. I Congreso de Petróleo y Gas, La Habana, Cuba.

Martínez-Rojas, E.; Morales-González, J. (2020). Interpretación sísmica de la zona de Oeste de Ceballos en el sector noreste de la Cuenca Central, Cuba. Centro de Investigaciones del Petróleo.
Mironov, V.S. (1977). Curso de prospección gravimétrica. Reverté.

Mondelo, F.; Sánchez, R. (2011). Mapas geofísicos regionales de gravimetría, magnetometría, intensidad y espectrometría gamma de la República de Cuba, escalas 1:2000000 hasta 1:50000. Instituto de Geología y Paleontología.

NASA/GSFC. (2020). Imagen, foto satélite de Cuba. http://mapas.owje.com/satellite/19/mapa-satelitecuba.php

Pardo-Echarte, M.E.; Rodríguez, O. (2016). Unconventional Methods for Oil \& Gas Exploration in Cuba. Springer.

Pardo-Echarte, M.E.; Rodríguez, O.; Delgado, O. (2019). Non-seismic and Non-conventional Exploration Methods for Oil and Gas in Cuba. Springer. https://doi.org/10.1007/978-3-03015824-8

Peña, A. (2005). Modelación 3D de datos gravimétricos del norte de la Cuenca Central. Tesis, Instituto Superior Minero Metalúrgico "Dr. Antonio Núñez Jiménez". Cuba.

Sterling, N.; Martínez-Rojas, E. (2018). Informe del procesamiento sísmico 2D de PSTM en líneas perteneciente a los bloques 21 y 23 de la Cuenca Central adquiridas en los años 1992, 1994 y 2004. Centro de Investigaciones del Petróleo.

Fecha de recibido: 13 de abril de 2021

Fecha de aprobado: 19 de noviembre de 2021 